\title{
Thinking on Training Strategies for Meta Learning Ability of Piano Learners at Higher Normal Schools
}

\author{
Jing LV \\ School of Music and Dance, Beifang Univesity of Nationality, Yinchuan, 750021, China
}

Keywords: Piano teaching at higher normal schools. Meta learning. Art of music. Learning methods

\begin{abstract}
Theories related to researches on piano teaching at higher normal schools, which has drawn much attention from the society, emerge endlessly. Usually, teaching implementers, teaching methods and teaching content are key points of scholars' researches. On the contrary, importance attached to researches on methods by which students learn piano at higher normal schools is not enough. This thesis will start with aspects, such as plans, evaluation, adjustment and summarization etc., in learning process, explain basic connotation of learning and analyze ways to cultivate meta learning ability of piano learners at higher normal schools.
\end{abstract}

\section{Introduction}

Development of social economy proposes higher requirements for talent and drives reform of teaching modes and methods of our higher education. Up to now, China has obtained some prominent research results in piano teaching reform at higher normal schools. Based on traditional one-to-one teaching method, grouping teaching is added and the method that the number of piano songs is increased is used to cultivate students' ability to accompany and give an instrumental performance further. Practices, for instance, higher normal schools add lessons about musical art appreciation to piano teaching, are content of researches on teaching reform of such schools. Indeed, such innovation in the aspect of teaching promotes piano teaching at higher normal schools. Piano teaching at higher normal schools not only directs at making students master related musical knowledge and playing skills but also aims at enabling students to grasp ways to learn piano at higher normal schools. Learners are subjects of education, so studies on how to improve students' independence in piano teaching at higher normal schools appear to be quite important. Because there are few researches on students' learning methods in piano teaching at higher normal schools, the following content will combine with latest research achievements of psychology, i.e., meta learning, to discuss learners' meta learning methods in piano teaching at higher normal schools.

\section{Connotation of meta learning}

\section{Generation of meta learning}

It was American psychologists that proposed the academic opinion meta learning in researches in 1976 for the first time. The said metacognition means people cognize objective world of their cognition, i.e., they uses the process in which the objective world is cognized as a target of cognition, which is different from the situation that we regard the objective world as an objective of cognition. According to concept of metacognition, psychological circle put forward the theory meta learning in 1993. Meta learning involves that learners can exert autonomy sufficiently in learning, make learning objectives according to their own situations, realize learning achievements are decided by different learning methods and learn the learning methods they adopt at present fully and master their own psychological trend in learning. At the same time, they are able to summarize the learning methods they apply to learning, rethink about advantages and disadvantages of their learning methods, find the ones that are appropriate for themselves, make learning plans according to learning objectives reasonably and arrange time and learning methods according to study plans appropriately . 


\section{Researches and development of meta learning theory in China}

\section{Analyzing content of meta learning according to factor analysis}

In accordance with researches of related Chinese scholars (represented by Dong Qi and Zhou Yong), they use factor analysis to divide meta learning into the following aspects; firstly, self-supervision before learning, i.e., plan and arrange learning schedule well, and prepare related tools needed by learning; secondly, self-supervision in the learning process, i.e., formulate learning objectives according to content that they will learn, choose appropriate learning methods to study specifically, implement learning plans that they have made before in learning and ensure effective implementation of learning; thirdly, self-supervision after finishing learning, i.e., summarize their mentality in the learning process, check whether application of learning methods is appropriate or not and whether learning achievements meet expected goals, find their disadvantages and advantages in learning via thinking about these aspects, consider ways to remedy such disadvantages and accumulate experience in learning simultaneously.

\section{Based on achievements in foreign countries}

On the basis that some scholars represented by Zhang Qinglin absorb researches achievements about meta learning in foreign countries, they summarize elements involved in meta learning ability, i.e., establish learning objectives according to practical situations, choose appropriate learning methods, be good at summarizing, finding and remedying disadvantages and adjust learning status and methods according to practical learning states.

\section{Summarization}

According to the foregoing research achievements, it can be concluded that meta learning means learners formulate learning objectives in accordance with their own situations, choose appropriate learning methods based on learning content, enhance evaluation and supervision on their mental conditions and learning methods in the learning process, adjust their mental status and learning methods to the best point in accordance with practical learning situations and improve both learning ability and learning effect.

\section{Ways to improve students' metal learning ability in piano teaching at higher normal schools}

\section{Enhancing students' metacognitive ability and improving their learning effect}

The said metacognition means students make cognitive objectives and choose appropriate cognitive methods according to content of cognition, adjust their cognitive methods in accordance with practical situations, ensure flexible and diversified learning methods are applied to cognitive activities effectively, strengthen pertinence of cognition and enhance motivation of independent study to improve effect of cognition and ensure successful implementation of cognitive activities. Meta learning theory is a teaching theory proposed based on metacognition theory. Thus, it is essential to enhance cultivation of students' metacognitive ability, i.e., target of students' cognition is the whole process in which they learn knowledge related to piano and quality of piano teaching at higher normal schools is improved by cultivating students' metacognitive ability. Currently, there are three common teaching situations existing in piano teaching at higher normal schools. Firstly, teachers do not permeate cultivation of students' metacognitive learning ability into teaching but deem that students can improve metacognitive ability by themselves and improvement in metacognitive ability depends on students themselves largely. Secondly, the method that breaks away from intensive training of subjects is used to train students' metacognitive ability in practical teaching. Thirdly, students improve their metacognitive ability gradually by teachers' occasional guidance and piano teachers with abundant teaching experience guide students to learn in constant introspection by virtue of their teaching level. All of the three teaching phenomena have defects. In detail, the first teaching phenomenon ignores cultivation of students' metacognitive ability in teaching, the second teaching phenomenon separates close connection between metacognition and subject learning actually, and the third teaching phenomenon still stays in an exploratory stage and has little effect on cultivation of students' metacognitive ability because it has no systematic and comprehensive training plans. Therefore, we should make scientific, systematic and complete plans about cultivation 
of students' metacognitive ability according to practical circumstances in teaching and include cultivation of metacognitive ability in the list of teachers' teaching objectives.

Cultivating students' ability to establish learning objectives that are suitable to actual circumstances in teaching

The situation that students establish an appropriate learning objective has some guiding effect on their learning and drives them to walk towards this objective in the learning process. Cultivation of students' ability to establish learning objectives in piano teaching at higher normal schools can be carried out according to the following steps. First of all, build short-term and long-term learning objectives, i.e., set learning objectives and make learning plans according to practical learning situations (students' psychological characteristics, learning status, learning ability and requirements for themselves). Since four-year college life is a learning process with a long cycle, hierarchical establishment of learning objectives accords with people's mental and physiological characteristics. Divide the four-year college life into semesters, regard piano learning in each semester as a medium-term learning goal, treat the four-year piano learning at colleges as a long-term learning goal and use weekly piano learning as a short-term goal. The medium-term and long-term learning goals can be realized if students realize the short-term goals they have established in learning constantly. Secondly, choose appropriate learning methods and learning auxiliary tools according to learning objectives. Because differences in learning content and learning materials decide that students' learning methods in the learning process cannot be the same all the time, it is necessary to choose learning methods that are appropriate for current learning content. For example, when new musical works are taught, teachers will ask students to collect related materials and learn various kinds of background under which such works are created (such as social background under which works are created, creating styles and features of music in the same period as such works as well as authors' background and writing styles etc.) to help students learn connotation of works. Then, teachers ask students to read musical scores by the standard that students must do well in give aspects (musical scale, fingering, performance method, expression during performance and playing rhythm) in order to make full preparation before learning. At the same time, teachers guide students to master ways to overcome technical difficulty as well as understand styles expressed by works and methods that can be used to improve performance level. The third step means that students judge whether their learning achievements can meet the learning objective that they have established according to their own learning status and adjust ways to play the piano and plans based on learning objectives. The fourth step involves summarization of the whole learning process. Teachers emphasize importance of after-school summarization in teaching repeatedly and guide students to find disadvantages existing in their piano study in teaching, think about remedying approaches, accumulate their advantages, rethink about their learning methods and perfect their own learning methods by the foregoing methods.

\section{Developing students' habit of summarizing study in piano teaching}

Whether learning effect is good or bad has close relationship with whether students are good at summarizing or not. Consequently, summarization of study appears to be quite important. To improve students' piano learning effect and enhance their skills used to play the piano, it is essential to ask students to do introspection and summarization in teaching constantly. Activities related to introspection and summarization should run through the whole learning process. Specifically, students should think about whether establishment of learning objectives is reasonable and effective or nor before building them, consider whether application of learning methods is appropriate and psychological state is good or not and make corresponding adjustment in the learning process, and carry out introspection and summarize disadvantages and successful experience after learning has ended. This is helpful for students to form piano learning methods that are appropriate for themselves. Therefore, effect of summarization can be enhanced in three aspects.

Firstly, teachers should have a habit of summarizing teaching in teaching process, summarize disadvantages and defects in teaching, look for remedying methods and improve teaching quality gradually. Teachers can help students develop a habit of summarizing only when they have developed summarizing habit in teaching. At the same time, teachers should enhance communication 
and exchange with students, which can not only help them supervise students' supervision in learning but also provide reference for teachers to learn students' ideological status by communication and enable teachers to have a definite object in teaching in order that the objective that teaching benefits teachers and students can be realized.

Secondly, platforms are provided for summarization and communication in teaching, i.e., some time is left for students to summarize their study in classroom to make students and teachers can communicate with one another and implement summarization during this period. In detail, a short period of time is reserved for teachers and students to have discussion. In this way, students' subjective initiative in the aspect of study can be exerted sufficiently and all teachers and students share experience in success and their shortages to learn from others' strong points and offset their own weakness on the premise that students play a dominant role and teachers offer guidance. Teachers hold an authoritative position, act as the center of teaching activities and pay more attention to imparting knowledge so that they explain knowledge in the whole classroom in traditional piano teaching. Thus, it is necessary to change modes of piano teaching at higher normal schools, build efficient classroom, exert democracy sufficiently, regard improvement in students' self-regulation of learning as a teaching requirement and use all kinds of ways to encourage students to think about and summarize their study in practical teaching in order to make them be good at finding problems, and dare to ask questions and express their own opinions. Meanwhile, teachers should evaluate students' answers and ideas appropriately. When students' opinions are good, have theoretical support but are different from teachers', teachers should encourage them in time. When students' opinions are good but different from playing skills, teachers ought to guide students to find disadvantages and find ways to solve problems.

At last, record all kinds of summarization in order that students can use them for reference conveniently in future. As the saying goes, good memory is not as good as bad writing. Changing oral experience into written records is helpful for students to improve their ability to give piano performance constantly. For example, students may summarize and conclude basic knowledge like introduction to recorded works, social background under which works are created, creating styles and features of music in the same period as such works as well as authors' background and writing styles, playing skills of works and difficult and key points in works. At the same time, students may record the content that teachers teach in classroom, their own experience in learning and disadvantages in study. According to such content planning, students can carry out learning plans in the next period of time, improve their skills in piano performance and perfect ways to learn to play the piano.

\section{Perfecting evaluation system of piano teaching}

In traditional teaching, teachers usually use examinations to evaluate students' learning achievements and master their learning situations. However, traditional teaching methods can only make teachers learn situations about students' mastery of knowledge. As a result, effective methods by which teachers can learn students' ability and techniques are lacked. Thus, teachers should encourage students to do self-evaluation in piano teaching at higher normal schools and students evaluate their learning situation by virtue of teachers' guidance, which can not only promote students' independent study but also help them improve their ability in self-evaluation. Content of students' self-evaluation should include whether establishment of learning objective is reasonable or not, whether application of learning methods is appropriate or not and whether learning walks towards learning objectives. Consequently, teachers should use flexible and diversified teaching methods to improve students' self-evaluation ability in piano teaching at higher normal schools in order to meet teaching objectives of students' meta learning in piano teaching at higher normal schools.

\section{Conclusion}

Exerting students' self-regulation of learning sufficiently and paying attention to students' personalized education are advocated by modern educational concept. Allowing students to be masters of study in piano teaching at higher normal schools can not only improve students' learning 
effect but also make them master ways to learn piano at higher normal schools. Meta learning accords with both modern educational concept and requirements of piano teaching reform at higher normal schools. Consequently, it is necessary to combine with piano teaching at higher normal schools with meta learning theory to enable students master methods used to learn knowledge about piano when they master knowledge related piano and lay a solid foundation for students' future study and work.

\section{References}

[1] Liu Xin and Yin Aiqing: Exploration on Investigative Study in Piano Course at Higher Normal Schools in China, Chinese Music, 2009(02).

[2] Wang Xiaohong: Training Strategies for Piano Learners' Meta Learning Ability at Higher Normal Schools, Hundred Schools in Art, 2010(06).

[3] Wang Xiaohong: On Training Strategies for Piano Learners' Independent Learning Ability at Higher Normal Schools, Hundred Schools in Art, 2012(01).

[4] Zheng Qian: Research on Effect and Practice of Metacognition in Piano Teaching at Higher Normal Schools [D]. Changsha: Hunan Normal University, 2012.

[5] Ge Dongyan: Research on Development of Piano Learning Ability of Students Majoring in Music Education at Higher Normal Schools and Countermeasures [D]. Suzhou: Suzhou University, 2013.

[6] Yuan Jin and Yuan Qin: Cultivation of Students' Learning Ability in Piano Learning at Higher Normal Schools, Jiangxi Education, 2010(Z6).

[7] Liang Yihong: Piano Teaching reform at Higher Normal Schools Under Education View of Sustainable Development, Musical Creation, 2011(3). 